\title{
Identification of Violent Response with Stretch Sensor Data from a Smart-Jacket using Naïve Bayes Algorithm
}

\author{
Princy Randhawa, Vijay Shanthagiri, Ajay Kumar
}

\begin{abstract}
In this paper, a smart-jacket using stretch sensors, pressure sensors was built for purpose of generating body-movements data and in order to record different kinds of signals and the distribution of the same on the jacket. Every degree of motion, when exercised, generates voltage changes in the stretch sensors as it is its property to do so. This data is collected in a flora chip set, which is Arduino based. The collected data is processed, pruned and filtered for outliers. This paper concerns with a supervised learning algorithm called Naive Bayes, which is applied over independent datasets, meaning one set of observation has no direct relations to each other. The placement of sensor are on the shoulders and elbows and the responses from each are independent of each other. Using Naive Bayes, the date has been classified for the violent response and the normal action.
\end{abstract}

Keywords: Nä̈ve Bayes, Physical Violence, Stretch Sensors, Smart jacket.

\section{INTRODUCTION}

It is quite obvious that during an accident situation the victim often goes out of control. An act of assault on a victim, which is also a kind of accident, renders the victim helpless. This issue becomes more serious and it is an alarming one if the victim is a woman (Randhawa et al., 2017). There are many self-protection weapons are currently available for women in situations like rape, assaults like anti rape belt, Pepper spray, Ninja Key chain, Hand gun etc.(Koshmak et al., 2013). Once the victim is attacked by the culprit, the victim is over powered after a short resistance by the offender. During this short struggle for escape, the fabric worn by the victim undergoes various degrees of stretching and compression. To achieve this, a generic jacket is envisioned on which Fabric sensors are woven on strategic positions in the jacket so as to record stretching, bending \& impact. Generally it has been observed that there will be certain degree of bending, stretching and impact on the body during normal action performed by the individual (Poupyrev et al., 2016). The identification of the normal data to that of data acquired under violent attack is a challenging task. The solution lies in strategically collecting the fabric sensor data when there is a certain degree of stretching and bending and collect the date for different intervals. The first requirement was a jacket in which various fabric sensors woven at strategic locations from where physical movements could be recorded. Data are

\footnotetext{
Revised Manuscript Received on November 08, 2019.

* Correspondence Author

${ }^{1}$ Princy Randhawa, ${ }^{2}$ Vijay Shanthagiri*, ${ }^{3}$ Ajay Kumar *

${ }^{1}$ princyrandhawa23@gmail.com, Manipal University Jaipur, India

2vijay.shanthagiri@gmail.com, Oeuvre Bangalore, India

3 ajay.kumar@jaipur.manipal.edu, Manipal University Jaipur, India
}

analysed acquired from body-worn sensors such as pressure sensors stretch sensor and accelerometer, as they were recorded in the dataset and it gives the data in the form of voltage fluctuations while stretching and bending (Randhawa et al., 2018). To achieve the same, an in house smart jacket was built as shown in the Figure 1 and Table 1 Shows the location of these units.
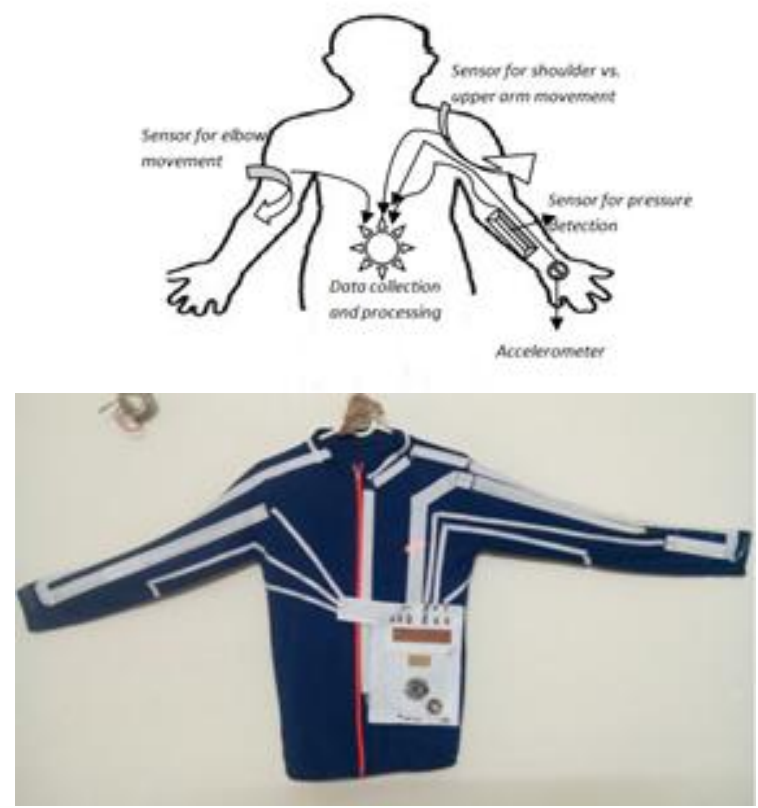

Figure 1: Custom Built Smart Jacket with placement of sensor

Table 1: Placement of Sensor

\begin{tabular}{|c|c|c|c|}
\hline & Accelerometer & $\begin{array}{c}\text { Stretch } \\
\text { Sensor }\end{array}$ & $\begin{array}{c}\text { Pressure } \\
\text { Sensor }\end{array}$ \\
\hline $\begin{array}{c}\text { Right } \\
\text { Elbow }\end{array}$ & $\mathbf{0}$ & 1 & 0 \\
\hline $\begin{array}{c}\text { Left } \\
\text { Elbow }\end{array}$ & $\mathbf{0}$ & $\mathbf{1}$ & 0 \\
\hline $\begin{array}{c}\text { Right } \\
\text { wrist }\end{array}$ & $\mathbf{0}$ & 0 & 1 \\
\hline $\begin{array}{c}\text { Left } \\
\text { wrist }\end{array}$ & $\mathbf{0}$ & 0 & 1 \\
\hline Hand & $\mathbf{1}$ & 0 & 0 \\
\hline
\end{tabular}

Our objective is to extract the data from the best training samples that enable the classification of the violent response and normal action of the user-dependent models. 


\section{Identification of Violent Attack using Algorithm}

The research focuses on predicting those actions which could be prove to be an animated out of force and not by normal action. For this we would need to collect ample motion data pertaining to specific parts of the body. Using that dataset, model is implemented using naïve Bayes classifier used for classification of the statistical data. It is named after Thomas Bayes, who proposed the Bayes Theorem. It is a classification technique based on Bayes' Theorem with an assumption of independence among predictors. A Naive Bayes classifier assumes that the there is no relation of one particular feature in a class to any other feature in a class (Zemp et al., 2016). This algorithm works on conditional probability. Using the conditional probability, we can calculate the probability of an event using its prior knowledge.

$$
P(\mathrm{H} \mid \mathrm{E})=\frac{P(\mathrm{E} \mid \mathrm{H}) * P(H]}{P(E)}
$$

- $\quad P(H)=$ The probability of hypothesis $\mathrm{H}$ being true. This is known as the prior probability.

- $\quad P(E)=$ The probability of the evidence.

- $\quad P(E \mid H)=$ The probability of the evidence given that hypothesis is true.

- $\quad P(H \mid E)=$ The probability of the hypothesis given that the evidence is there.

The custom built stretch sensors used to achieve reliable sensitivity to stretching and bending that would result during motion. The different kinds of fabric-buslines were created to interconnect all the devices used in the jacket. The jacket is wired using conductive material and conductive thread, and the voltage change is read by Flora chipset wired to all the stretch sensors as shown in Figure 2
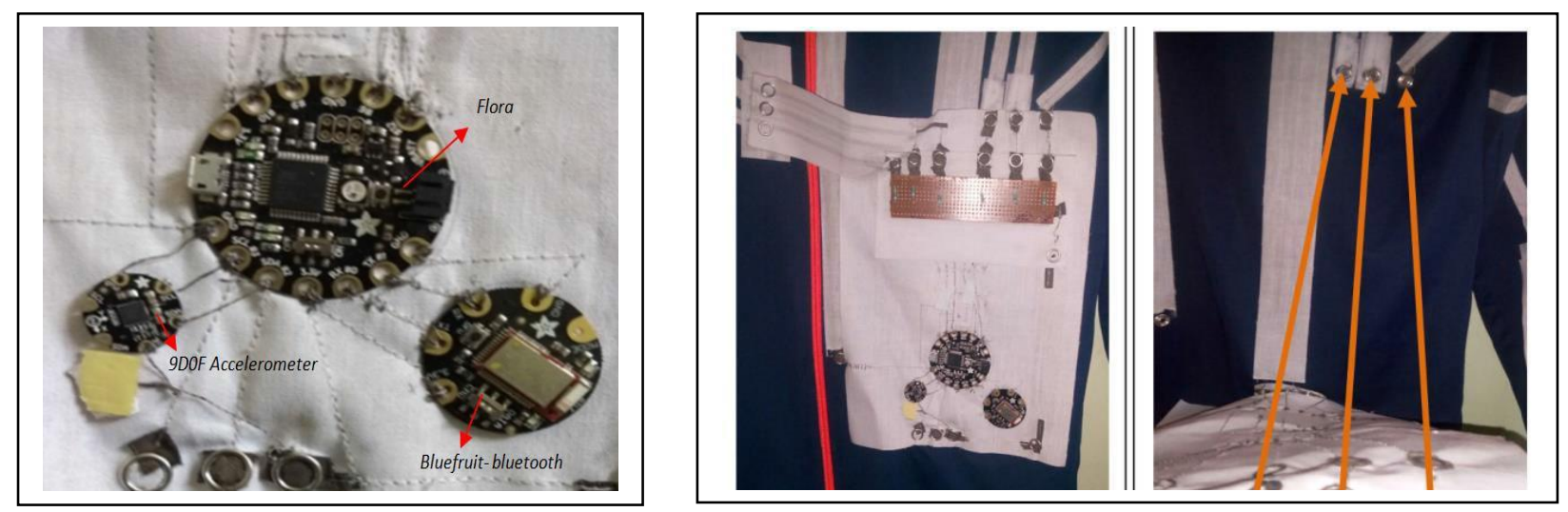

Figure 2: Flora chip attached to the stretch sensors

Consider Table 2 which contains an instance of the data recorded from the sensors attached to the jacket. PR \& PL contains the data recorded from the pressure sensor of the right and left hand with different probabilities. Similarly ER \& EL elbow sensor with the probabilities.

\section{Table 2: Fabric Sensor Data}

\begin{tabular}{|l|l|l|l|l|l|l|l|}
\hline PR & P & PL & P & ER & P & EL & P \\
\hline 35 & 0.1 & 36 & 0.1 & 33 & 0.1 & 37 & 0.1 \\
\hline 35 & 0.1 & 32 & 0.1 & 31 & 0.1 & 35 & 0.1 \\
\hline 33 & 0.1 & 24 & 0.1 & 31 & 0.1 & 33 & 0.1 \\
\hline 33 & 0.1 & 24 & 0.1 & 24 & 0.1 & 33 & 0.1 \\
\hline 32 & 0.1 & 24 & 0.1 & 24 & 0.1 & 33 & 0.1 \\
\hline 32 & 0.1 & 19 & 0.1 & 19 & 0.1 & 32 & 0.1 \\
\hline 28 & 0.1 & 19 & 0.1 & 19 & 0.1 & 32 & 0.1 \\
\hline 28 & 0.1 & 19 & 0.1 & 14 & 0.1 & 28 & 0.1 \\
\hline 24 & 0.1 & 14 & 0.1 & 14 & 0.9 & 28 & 0.1 \\
\hline 24 & 0.1 & 14 & 0.1 & 14 & 0.9 & 28 & 0.1 \\
\hline 19 & 0.1 & 11 & 0.1 & 8 & 0.9 & 24 & 0.1 \\
\hline 19 & 0.1 & 11 & 0.1 & 8 & 0.9 & 24 & 0.1 \\
\hline 14 & 0.9 & 8 & 0.9 & 8 & 0.9 & 24 & 0.1 \\
\hline 14 & 0.9 & 8 & 0.9 & 8 & 0.9 & 24 & 0.1 \\
\hline 11 & 0.9 & 8 & 0.9 & 8 & 0.9 & 14 & 0.1 \\
\hline 11 & 0.9 & 8 & 0.9 & 8 & 0.9 & 14 & 0.1 \\
\hline
\end{tabular}

As we have observed in Table 1 , the value gathered when the sensors are not activated is 35 . This is a value as recorded from the sensor under normal stable motionless state. This value changes when pressure is applied on the sensor located on the right forehand. The value gradually decreases to minimum 


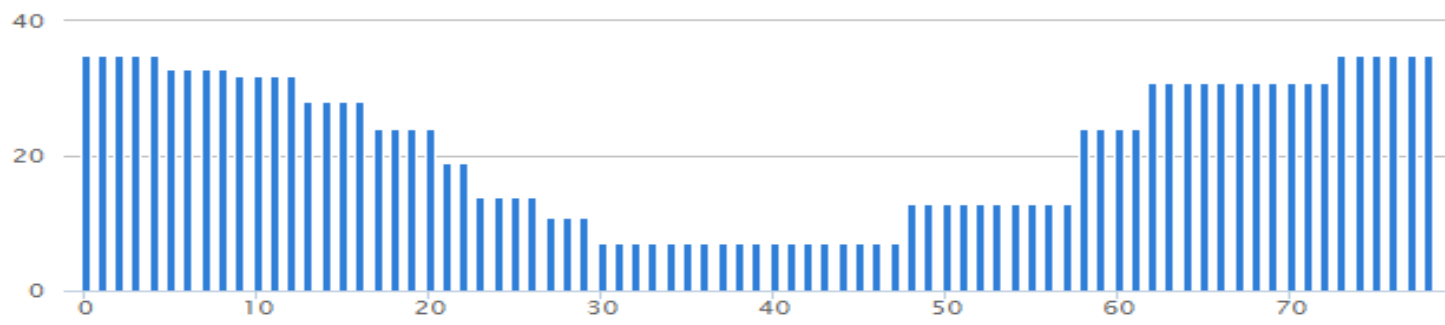

Figure 3. Graphical view of column PL sample (Right hand pressure sensor)

The same is true for PR, ER and EL. To apply Naive-Bayes Algorithm, need to establish the accuracy of the data. The decrease in value of the sensor from 35 to 7 happened due to pressure applied on the sensor, it shows that the probability of hand being pressed would be 0.9 . Hence any decrease in the value from 35 until 7 will all have a probabilistic value for the pressing of hand. To establish a threshold to be accurate that the hand was indeed pressed, normalize the value.

Table 3: Statistical calculations

Table 3: Statistical calculations
\begin{tabular}{|l|l|}
\hline Sample Standard Deviation, $\mathrm{s}$ & 10.891 \\
\hline Variance (Sample Standard), $\mathrm{s}^{2}$ & 118.634 \\
\hline Population Standard Deviation,$\sigma$ & 10.822 \\
\hline Variance (Population Standard), $\sigma^{2}$ & 117.133 \\
\hline Total Numbers, N & 79 \\
\hline Sum : & 1673 \\
\hline Mean : & 21.177 \\
\hline Standard Error of the Mean $\left(S E_{\hat{\alpha}}\right):$ & 1.2254 \\
\hline
\end{tabular}

Following are the calculations for the sample of Column A only. Since the distribution is a normal distribution, assuming at this initial stage that there won't be any outliers in the sample. Confidence interval approximations assuming that the sample will always follow a normal distributions can be found below.

\begin{tabular}{|c|c|}
\hline Confidence Level & Range \\
\hline $68.3 \%, S E_{\bar{X}}$ & $19.951-22.402$ \\
\hline $90 \%, 1.645 S E_{\bar{\gamma}}$ & 19.161-23.193 \\
\hline $95 \%, 1.960 S E_{\hat{z}}$ & $18.775-23.579$ \\
\hline $99 \%, 2.576 S E_{\hat{X}}$ & $18.020-24.333$ \\
\hline $99.9 \%, 3.291 S E_{\hat{X}}$ & $17.144-25.210$ \\
\hline $99.99 \%, 3.891 S E_{\hat{X}}$ & $16.409-25.945$ \\
\hline $99.9999 \%, 4.4877 S E_{\hat{X}}$ & $15.764-27.172$ \\
\hline
\end{tabular}

It would have been easy to set the mean as the threshold value, but when we observe Figure 4 we can see that $99.9999 \%$ accuracy is achieved at 4.9 standard error of mean. The range at this value is 15 to 27 . The Table 2 shows the mean of the sample is 21 . By keeping the confidence level in view, we can set 15 as the threshold of the value. It means that any time the value of the sensor goes below 15, we can be $99.99 \%$ sure that the hand has been pressed so every value above 15 will be tagged with 0.1 and every value below 15 will be tagged with 0.99 . The above process is followed for each of the column. Since all the columns are following a normal distribution, we can assume that 15 can be set as a threshold or lower maximum. After appending the probabilities the new table would look as shown in Table 5 with all the four sensors activated.

Table 2: Fabric Sensor with Probabilities
\begin{tabular}{|l|l|l|l|l|l|l|l|}
\hline PR & P & PL & P & ER & P & EL & P \\
\hline 35 & 0.1 & 36 & 0.1 & 33 & 0.1 & 37 & 0.1 \\
\hline 33 & 0.1 & 36 & 0.1 & 33 & 0.1 & 37 & 0.1 \\
\hline 32 & 0.1 & 28 & 0.1 & 24 & 0.1 & 35 & 0.1 \\
\hline 32 & 0.1 & 24 & 0.1 & 19 & 0.1 & 35 & 0.1 \\
\hline 28 & 0.1 & 24 & 0.1 & 14 & 0.9 & 33 & 0.1 \\
\hline 33 & 0.1 & 19 & 0.1 & 14 & 0.9 & 33 & 0.1 \\
\hline 32 & 0.1 & 14 & 0.9 & 8 & 0.9 & 32 & 0.1 \\
\hline 28 & 0.1 & 14 & 0.9 & 8 & 0.9 & 32 & 0.1 \\
\hline 28 & 0.1 & 8 & 0.9 & 6 & 0.9 & 28 & 0.1 \\
\hline
\end{tabular}




\begin{tabular}{|l|l|l|l|l|l|l|l|}
\hline 24 & 0.1 & 8 & 0.9 & 6 & 0.9 & 28 & 0.1 \\
\hline 24 & 0.1 & 8 & 0.9 & 6 & 0.9 & 28 & 0.1 \\
\hline 19 & 0.1 & 7 & 0.9 & 7 & 0.9 & 24 & 0.1 \\
\hline 19 & 0.1 & 7 & 0.9 & 7 & 0.9 & 24 & 0.1 \\
\hline 14 & 0.9 & 7 & 0.9 & 7 & 0.9 & 24 & 0.1 \\
\hline 14 & 0.9 & 7 & 0.9 & 7 & 0.9 & 24 & 0.1 \\
\hline 11 & 0.9 & 12 & 0.9 & 12 & 0.9 & 14 & 0.9 \\
\hline 11 & 0.9 & 12 & 0.9 & 12 & 0.9 & 14 & 0.9 \\
\hline 7 & 0.9 & 12 & 0.9 & 12 & 0.9 & 7 & 0.9 \\
\hline 7 & 0.9 & 12 & 0.9 & 12 & 0.9 & 7 & 0.9 \\
\hline 13 & 0.9 & 12 & 0.9 & 12 & 0.9 & 12 & 0.9 \\
\hline 13 & 0.9 & 12 & 0.9 & 12 & 0.9 & 24 & 0.1 \\
\hline
\end{tabular}

To detect a violent attack, we need to define what a violent attack is. The table below is created to hypothesize an attack. If just a hand is pressed will it be a violent attack on a person • The answer could be yes or no. But considering the fact tha we have four sensors to measure activity, and only one senso is showing an activated state, can say that pressing of just th pressure sensor on the hand could be assumed as the mea] average of the normalized value of four sensors.

Using our dataset, the Naive Bayes concept can be explained as:

- Let's assume that no pair of features are dependent. E.g., the Right pressure sensor being 'being stretched' doesn't mean that the person is being attacked or the right elbow being bent does not proved of attack sequence. Hence, all the features are assumed to be independent.

Each feature is given the same weight. E.g., only knowing the value of right pressure sensor and right elbow sensor cannot predict the outcome accurately. Assuming all the features are contributing equally to the outcome and cannot say that that any attribute is relevant to the outcome.

This means that if the record in the table 6 is an instance of the data recorded, then the chance that the person is under attack is 0.32.It means there are less chances of the person being attacked because it can be a normal motion also so cannot be predicted whether actually the person is being attacked or not

Table 6: Fabric sensor threshold probabilities with Different sensors activated

\begin{tabular}{|l|l|l|l|l|}
\hline PR & PL & ER & EL & Mean \\
\hline 0.99 & 0.1 & 0.1 & 0.1 & 0.3225 \\
\hline 0.99 & 0.99 & 0.1 & 0.1 & 0.54 \\
\hline 0.99 & 0.99 & 0.99 & 0.99 & 0.99 \\
\hline
\end{tabular}

From column 3, we can note that to be assured of a violent attack if all the sensors are activated. It means the all the actions performed out of force and not of normal action. Meaning a person's both hands are pressed and both elbows are bent because of agitation, then all four sensors are activated and a 99.9 percent accurate prediction of violent attack is observed. There are more chances that the person is being attacked rather than normal motion It will act as a deterrence if assaulter tries to o over powered the victim. To increase the accuracy the data is being trained for all the states of motion. For every set in the training data, the mean average is calculated to supervise the Naive-Bayes algorithm in learning the pattern. Table 8 shows the training dataset and testing dataset after being trained to know the chances of probability that the person is being attacked or not.

.Table 6: Training and Testing Set

\begin{tabular}{|c|c|c|c|c|}
\hline PR & PL & ER & EL & Mean \\
\hline 0.1 & 0.1 & 0.1 & 0.1 & 0.1 \\
\hline 0.1 & 0.1 & 0.1 & 0.1 & 0.1 \\
\hline 0.1 & 0.1 & 0.1 & 0.9 & 0.325 \\
\hline 0.1 & 0.1 & 0.1 & 0.9 & 0.325 \\
\hline 0.9 & 0.1 & 0.1 & 0.1 & 0.325 \\
\hline 0.9 & 0.9 & 0.1 & 0.1 & 0.54 \\
\hline 0.9 & 0.1 & 0.9 & 0.1 & 0.54 \\
\hline 0.1 & 0.1 & 0.9 & 0.9 & 0.54 \\
\hline 0.1 & 0.9 & 0.1 & 0.1 & 0.325 \\
\hline 0.1 & 0.1 & 0.1 & 0.1 & 0.1 \\
\hline
\end{tabular}

\begin{tabular}{|c|c|c|c|c|}
0.1 & 0.9 & 0.9 & 0.9 & 0.76 \\
\hline 0.1 & 0.9 & 0.9 & 0.9 & 0.76 \\
\hline 0.9 & 0.9 & 0.9 & 0.1 & 0.76 \\
\hline 0.9 & 0.9 & 0.1 & 0.1 & 0.54 \\
\hline 0.9 & 0.9 & 0.9 & 0.9 & 0.9 \\
\hline 0.9 & 0.9 & 0.9 & 0.9 & 0.9 \\
\hline 0.1 & 0.9 & 0.9 & 0.9 & 0.9 \\
\hline
\end{tabular}

After application of naive-bayes algorithm, we obtained the output as shown in the Table 7. A graphical view of the result obtained would give a much better perspective over the results obtained so as to facilitate intuitive judgment about the accuracy of the prediction. A graphical view of the result obtained would give a much better perspective over the results obtained so as to facilitate intuitive judgement about the accuracy of the prediction. After application of naive-bayes algorithm, we obtained the output as shown in the table 9. A graphical view of the result obtained would give a much better perspective over the results obtained so as to facilitate intuitive judgment about the accuracy of the prediction as shown in figure 4 . The pictorial representation of the excitation values shown as a histogram enables us to identify and correlate the probabilities which are close to 1 when the excitation happens. 
International Journal of Innovative Technology and Exploring Engineering (IJITEE) ISSN: 2278-3075, Volume-9 Issue-1, November 2019

Table 7: Prediction after classification

\begin{tabular}{|r|r|r|r|r|}
\hline PR & \multicolumn{1}{|l|}{ PL } & \multicolumn{1}{|l|}{ ER } & Prediction \\
\hline 0.9 & 0.9 & 0.9 & 0.1 & 0.76 \\
\hline 0.9 & 0.9 & 0.1 & 0.1 & 0.54 \\
\hline 0.9 & 0.9 & 0.9 & 0.9 & 0.9 \\
\hline 0.9 & 0.9 & 0.9 & 0.9 & 0.9 \\
\hline 0.1 & 0.9 & 0.9 & 0.9 & 0.9 \\
\hline 0.9 & 0.1 & 0.9 & 0.1 & 0.54 \\
\hline 0.1 & 0.1 & 0.9 & 0.9 & 0.54 \\
\hline 0.1 & 0.9 & 0.1 & 0.1 & 0.325 \\
\hline
\end{tabular}

\begin{tabular}{|r|r|r|r|r|}
\hline 0.1 & 0.1 & 0.1 & 0.1 & 0.1 \\
\hline 0.1 & 0.9 & 0.9 & 0.9 & 0.76 \\
\hline 0.1 & 0.1 & 0.1 & 0.1 & 0.1 \\
\hline 0.1 & 0.1 & 0.1 & 0.1 & 0.1 \\
\hline 0.1 & 0.1 & 0.1 & 0.9 & 0.325 \\
\hline 0.1 & 0.1 & 0.1 & 0.9 & 0.325 \\
\hline 0.9 & 0.1 & 0.1 & 0.1 & 0.325 \\
\hline 0.1 & 0.1 & 0.1 & 0.1 & 0.1 \\
\hline 0.1 & 0.1 & 0.1 & 0.1 & 0.1 \\
\hline
\end{tabular}

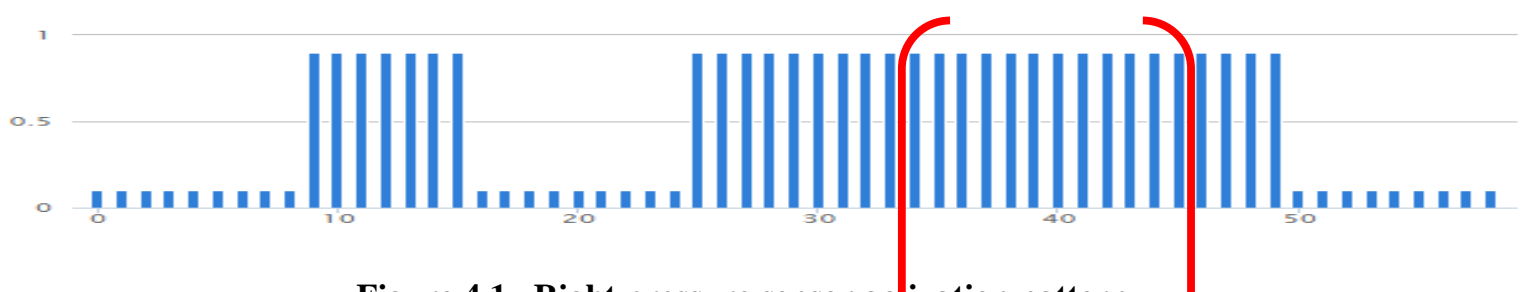

Figure 4.1. Right pressure sensor ac ivation pattern
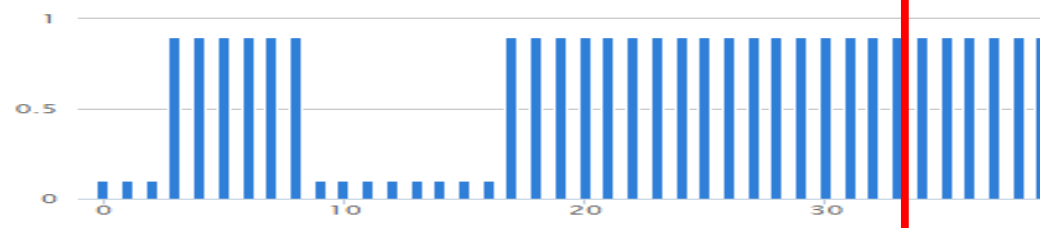

Figure 4.2. Left pressure sensor acti ation pattern
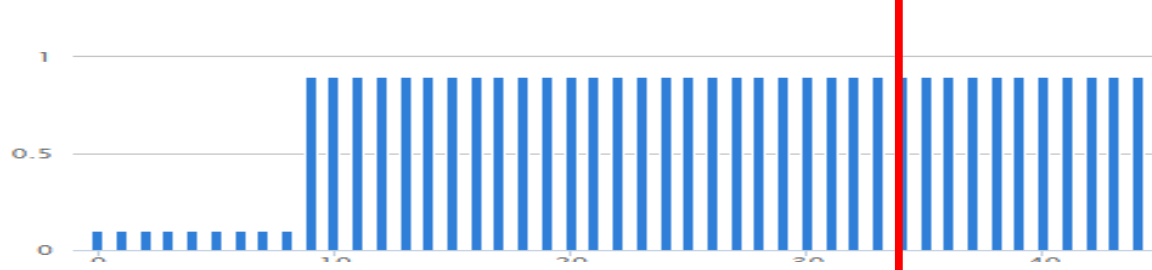

Figure 4.3. Right elbow sensor activation pattern

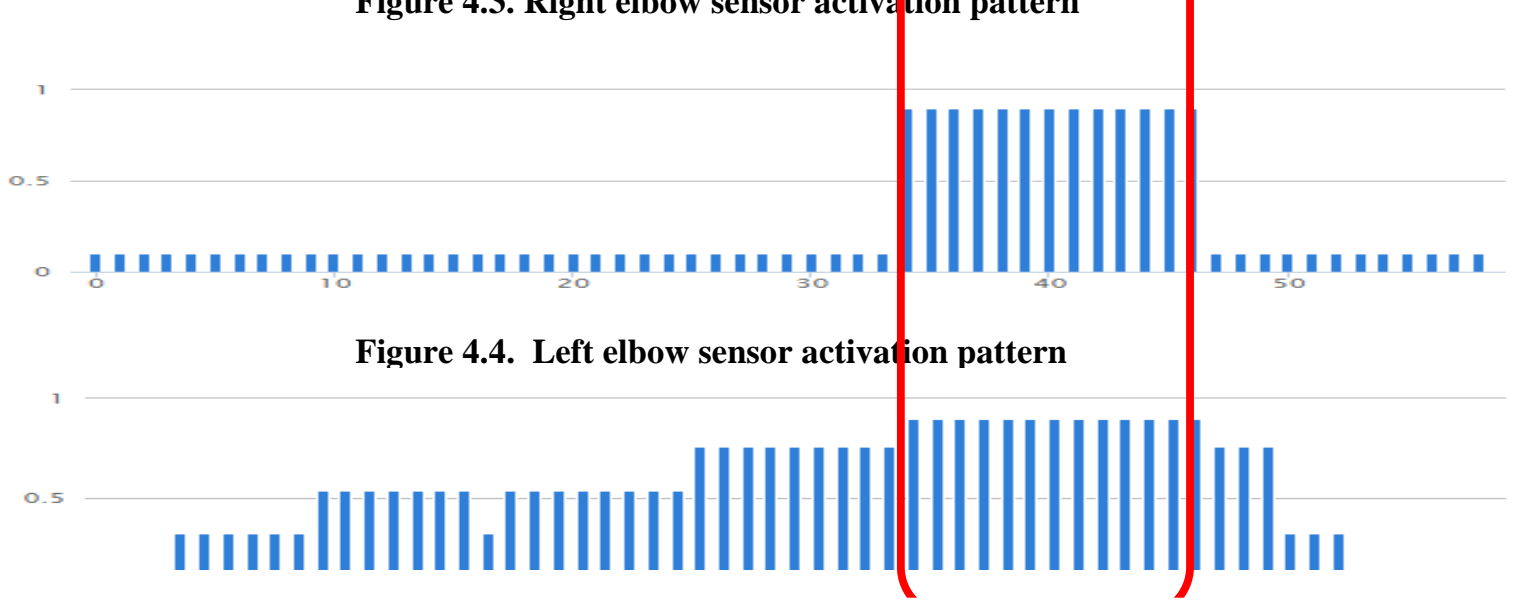

Figure 4. Histogram of sensor excitation table clearly depicting the areas where all the sensors are activated

\section{RESEARCH SCOPE}

The scope of study mainly pertains to identification of physical activities which involves distinct physical motion and physical reactions. Further, the study extends to identifying highly responsive fabric sensors for various reasons such as stretching of elbow and shoulders. The data collected from the sensors will be pre-processed with standard principles of machine learning so as to be effective in achieving successful classification. The scope of study as seen above extends broadly into physiological motion of human body. A detailed study of stretch sensors, fabric sensors, and wearable computational 
units such as flora microcontroller, accelerometers is required to select and implement the computational logic on the hardware. The scope also extends to Machine Learning and data pre-processing. Since the final result of the research is to develop a system to identify human motion during normal activities and panic driven responses, most of the research will be about how the data being collected is pre-processed, categorised and analysed using machine learning algorithms to achieve the results.

\section{SIGNIFICANCE OF THE STUDY}

The significance of this research can be drawn from the fact that it builds on established discipline of embedded electronics, fabric sensors and machine learning. The main goal of classification between normal physical activity and panic driven activity however has never been undertaken. It is a novel attempt to measure voltage fluctuations caused by physical motion. Machine learning has been applied to various set of data and each set presents its own challenges. We attempt to discover the problems that would be prevalent when stretch sensor data which is nothing but voltage fluctuation data is collected for a different parts of the body and is subjected to classification. The research, by nature of the desired goal, goes deep in its treatment of machine learning to fabric sensor data.

\section{CONCLUSION}

It can be concluded from the above results that Bayesian technique can be successfully and reasonable applied to stretch sensor data for position and orientation classification. It is also evident that Bayesian analysis fits well for such data and if properly observed using graphs, one can get a validation of the results as well. However in the above results it is still not clear if it was a violent activity alone which brought about the sensor excitation. Further improvements in the algorithm and extension in the algorithm could facilitate in answering distinctively that the cause of the voltage variation and the subsequent sensor excitation was indeed due to a simultaneous and an almost haphazard reactive motion by the human wearing the jacket.

\section{REFERENCES}

1. Cornacchia, M., Ozcan, K., Zheng, Y., \& Velipasalar, S. (2017). Using Wearable Sensors, 17(2), 386-403.

2. N. Noury, A. Galay, J. Pasquier, and M. Ballussaud, "Preliminary investigation into the use of autonomous fall detectors," in Proc. Int. Conf. IEEE Eng. Med. Biol. Soc., Aug. 2008, pp. 2828-2831.

3. C. C. Wang et al., "Development of a fall detecting system for the elderly residents," in Proc. 2nd Int. Conf. Bioinform. Biomed. Eng., 2008, pp. 1359-1362.

4. T. Tamura, "Wearable accelerometer in clinical use," in Proc. Int. Conf. IEEE Eng. Med. Biol. Soc., vol. 7, Jan. 2005, pp. 7165-7166.

5. Y. Cao, Y. Yang, and W. H. Liu, "E-FallD: A fall detection system using Android-based smartphone," in Proc. 9th Int. Conf. Fuzzy Syst. Knowl. Discovery, May 2012, pp. 1509-1513.

6. M. R. Narayanan, S. R. Lord, M. M. Budge, B. G. Celler, and N. H. Lovell, "Falls management: Detection and prevention, using a waist-mounted triaxial accelerometer," in Proc. 29th Annu. Int. Conf. IEEE Eng. Med. Biol. Soc. (EMBS), Aug. 2007, pp. 4037-4040

7. J. Mantyjarvi, J. Himberg, and T. Seppanen, "Recognizing human motion with multiple acceleration sensors," in Proc. IEEE Int. Conf. Syst., Man, Cybern., vol. 2, Oct. 2001, pp. 2-7

8. D. M. Karantonis, M. R. Narayanan, M. Mathie, N. H. Lovell, and B G. Celler, "Implementation of a real-time human movement classifier using a triaxial accelerometer for ambulatory monitoring," IEEE Trans. Inf. Technol. Biomed., vol. 10, no. 1, pp. 156-167, Jan. 2006

9. I. Mandal, S. L. Happy, D. P. Behera, and A. Routray, "A framework for human activity recognition based on accelerometer data," in Proc. 5th Int. Conf. Next Generat. Inf. Technol. Summit (Confluence), Sep. 2014, pp. 600-603.

10. C. Zhu and W. Sheng, "Human daily activity recognition in robotassisted living using multi-sensor fusion," in Proc. IEEE Int. Conf. Robot. Autom., May 2009, pp. 2154-2159

11. L. Gao, A. K. Bourke, and J. Nelson, "Evaluation of accelerometer based multi-sensor versus single-sensor activity recognition systems," Med. Eng. Phys., vol. 36, no. 6, pp. 779-785, Jun. 2014.

12. M. Yu, A. Rhuma, S. Naqvi, L. Wang, and J. Chambers, "Posture recognition based fall detection system for monitoring an elderly person in a smart home environment," IEEE Trans. Inf. Technol. Biomed., vol. 16, no. 6, p. 1, Nov. 2012.

13. Cheng, L., Power, K., Corporation, T., Guan, Y., Zhu, K., \& Li, Y. (2017). Learning Methods with Wearable Sensors.

14. S. H. Fang, Y. C. Liang, and K. M. Chiu, "Developing a mobile phonebased fall detection system on Android platform," in Proc. Comput., Commun. Appl. Conf., 2012, pp. 143-146.

15. J. Dai, X. Bai, Z. Yang, Z. Shen, and D. Xuan, "PerFallD: A pervasive fall detection system using mobile phones," in Proc. IEEE Int. Conf. Pervasive Comput. Commun. Workshops, Mar. 2010, pp. 292-297.

16. M. Shoaib, S. Bosch, O. D. Incel, H. Scholten, and P. J. M. Havinga, "A survey of Online activity recognition using mobile phones," Sensors, vol. 15, no. 1, pp. 2059-2085, 2015.

17. G. A. Koshmak, M. Linden, and A. Loutfi, "Evaluation of the Androidbased fall detection system with physiological data monitoring," in Proc. Annu. Int. Conf. IEEE Eng. Med. Biol. Soc. (EMBS), Jul. 2013, pp. 1164-1168.

18. X. Long, B. Yin, and R. M. Aarts, "Single-accelerometer-based daily physical activity classification," in Proc. Int. Conf. IEEE Eng. Med. Biol. Soc., Sep. 2009, pp. 6107-6110

19. M. Muehlbauer, G. Bahle, and P. Lukowicz, "What can an arm holster worn smart phone do for activity recognition?" in Proc. Int. Symp. Wearable Comput. (ISWC), 2011, pp. 79-82.

20. Preece, S. J., Goulermas, J. Y., Kenney, L. P. J., \& Howard, D. (2009). A Comparison of Feature Extraction Methods for the Classification of Dynamic Activities from Accelerometer Data, 56(3), 871-879.

21. Tang, W., \& Sazonov, E. S. (2014). Highly Accurate Recognition of Human Postures and Activities Through Classification With Rejection, 18(1), 309-315.

22. C. M. Bishop, Pattern Recognition and Machine Learning,vol.1, Springer, New York, NY, USA, 2006.

23. Zemp, R., Tanadini, M., Plüss, S., Schnüriger, K., Singh, N. B., Taylor, W. R., \& Lorenzetti, S. (2016). Application of Machine Learning Approaches for Classifying Sitting Posture Based on Force and Acceleration Sensors.

24. Y. Cao, Y. Yang, and W. H. Liu, "E-FallD: A fall detection system using Android-based smartphone," in Proc. 9th Int. Conf. Fuzzy Syst. Knowl. Discovery, May 2012, pp. 1509-1513.

25. Vaithiyanathan, V., Rajeswari, K., Tajane, K., \& Pitale, R. (2013). Comparison of different classification techniques, 6(2), 764-768.

26. Zerrouki, N., \& Houacine, A. (2014). Automatic Classification of Human Body Postures Based on the Truncated SVD, 2(1), 58-62. https://doi.org/10.7763/JACN.2014.V2.82

27. Randhawa, Princy \& Shanthagiri, Vijay \& Mour, Rishabh \& Kumar, Ajay. (2018). Design and Development of Smart-Jacket for Posture Detection. 1-5.

28. Randhawa, Princy \& Shanthagiri, Vijay \& Kumar, Ajay.. "A review on applied machine learning in wearable technology and its applications." 2017 International Conference on Intelligent Sustainable Systems (ICISS) (2017): 347-354.

29. Poupyrev, I., Gong, N.-W., Fukuhara, S., Karagozler, M. E., Schwesig, C., \& Robinson, K. E. (2016). Project Jacquard: Interactive Digital Textiles at Scale. Proceedings of the 2016 CHI Conference on Human Factors in Computing Systems, 4216-4227

30. Stoppa, M., \& Chiolerio, A. (2014). Wearable electronics and smart textiles: A critical review. Sensors (Switzerland), 14(7), 11957-11992 